\title{
Overexpression FAT4 \& Down Regulation NAT2 in Human Breast Cancer
}

\author{
Fatemeh Eshaghi ${ }^{1}$, Vajihe Zarrin Pour ${ }^{2, *}$ Alireza Abdollahi ${ }^{3}$, Hadis \\ Mohammadpour ${ }^{4}$
}

${ }^{1}$ Department of Genetics, Damghan Branch, Islamic Azad University, Damghan, Iran ${ }^{2}$ Department of Biology, Tehran Central Branch, Islamic Azad University, Tehran, Iran

${ }^{3}$ Pathology Department, Institute Cancer, Imam Hospital, Tehran, Iran

${ }^{4}$ Iran National Tumor Bank, Tehran Medical University, Iran

DOI: $10.21859 /$ mci-supp-66

${ }^{*}$ Corresponding Author: Vajihe Zarrin Pour, Department of biology, Tehran central branch, Islamic Azad University, Tehran, Iran.E-mail:mahsa_eshaghi@yahoo.com

\section{Keywords:}

Breast Cancer

Expression

Fat4

Nat2

\section{Abstract}

Introduction: Nowadays, cancer is known as one of the major problems of human society and scientists and researchers are looking for short and very effective ways to identify and treat this group of diseases. One of these ways to investigate specific genes in pathways that can be used to identify cancer-specific biomarkers, containing expression FAT4 and NAT2 in breast cancer and the effect on the signaling pathway in human cells. Because of the comparison high frequency of some FAT4 and NAT2 genotypes in the population, the Attributable cancer risk might be high. The effect of FAT4 and NAT2 genotype on cancer risk varies with organ location, maybe reflecting tissue-specific expression of FAT4 and NAT2. Materials and Methods: Here we took 25 tumor samples and adjacent normal tissue of Iran National tumor Bank, we found the up regulation FAT4 and down regulation NAT2. Results: There is no evidence for the relation between FAT4 and NAT2 $(\mathrm{P}>0.05)$.

Conclusions: The results propose there is an interaction between FAT4 genotype with P53 signaling pathway $(\mathrm{P}<0.05)$. 